\title{
Opióides na Dor Oncológica e o seu Uso em Circunstâncias Particulares: Uma Revisão Narrativa
}

\author{
Opioids for Cancer Pain and its Use under Particular \\ Conditions: A Narrative Review
}

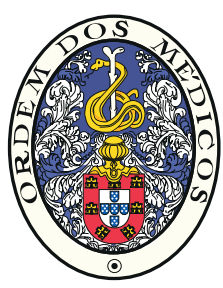

\author{
Cláudia VIEIRA $\triangle^{1,2,3}$, Marta BRÁS ${ }^{4}$, Maria FRAGOSO 1,5 \\ Acta Med Port 2019 May;32(5):388-399 - https://doi.org/10.20344/amp.10500
}

\section{RESUMO}

Introdução: A dor é equiparada a quinto sinal vital e deve ser avaliada de forma sistemática em todas as consultas de um paciente com cancro. Os fármacos utilizados na prática oncológica para tratar os doentes com dor crónica moderada a severa incluem analgésicos de ação periférica e central que têm sido abordados em múltiplas diretrizes nacionais e internacionais. No entanto, na prática clínica há que equacionar outros problemas e eventuais comorbilidades, que podem levantar dúvidas no momento da prescrição.

Material e Métodos: Fez-se uma revisão da literatura, tentando refletir sobre algumas situações específicas na utilização de opióides, nomeadamente perda da via oral, insuficiência renal (hemodiálise), insuficiência hepática, interações medicamentosas e formulações de ação imediata.

Resultados: Os opiáceos naturais (morfina e codeína) e os seus derivados sintéticos e semissintéticos (hidromorfona, oxicodona, fentanilo), o agonista parcial buprenorfina e finalmente o tramadol e tapentadol foram selecionados para esta análise. Os sistemas transdérmicos estão apenas disponíveis para a buprenorfina e o fentanilo. $\mathrm{Na}$ insuficiência hepática, o fentanilo foi considerado seguro, mas com exceção da codeína e do tramadol, podem todos ser usados com precaução. Na insuficiência renal, o fentanilo, a hidromorfona e o tapentadol foram considerados seguros. Deve evitar-se a morfina, e os restantes poderão ser usados com precaução. Em pacientes em hemodiálise pode usar-se buprenorfina, fentanilo, hidromorfona e tramadol (em doses até $200 \mathrm{mg} / \mathrm{dia}$ ).

Discussão: O não reconhecimento do impacto das várias situações descritas ao longo deste trabalho, nomeadamente a alteração da biodisponibilidade por perda de via oral, por alteração da farmacocinética e farmacodinâmica dos vários fármacos, quer no contexto da insuficiência de órgão responsável pelo metabolismo ou excreção, quer no contexto das interações farmacológicas, condiciona um norme risco de subtratamento da dor e consequente impacto em termos de qualidade de vida.

Conclusão: A prescrição de opióides é segura e efetiva, mesmo em situações de comorbilidades moderadas a graves como insuficiência renal e hepática e em doentes sem via oral disponível. Neste caso, como quando considerámos as interações farmacológicas, o plano terapêutico deve ser individualizado e o paciente deve ser avaliado regularmente. A seleção inadequada e/ou dose mal ajustada de um fármaco, o não reconhecimento do impacto dos efeitos adversos, frequentemente justificam o mau controlo da dor e a toxicidade excessiva.

Palavras-chave: Analgésicos Opioides/uso terapêutico; Dor Oncológica/tratamento; Insuficiência Hepática; Insuficiência Renal; Perturbações da Deglutição; Neoplasias

\section{ABSTRACT}

Introduction: All health professionals should be aware of the importance of evaluating pain - fifth vital sign- in cancer patients. Peripheral and central acting analgesics are widely used to treat moderate to severe pain, particularly cancer pain. Many guidelines have addressed this issue. However, real life patients' have other problems and comorbidities that may raise doubts when prescribing.

Material and Methods: Authors made a literature search, trying to clarify same specific situations: loss of oral route, renal impairment (hemodialysis), hepatic impairment, frequent opiod interactions and the availability of short-acting formulations.

Results: The following medicines were included in this analysis: the natural opiates (morphine and codeine), their synthetic and semisynthetic derivatives (hydromorphone, oxycodone, and fentanyl), the partial agonist buprenorphine and finally tramadol and tapentadol. Transdermal systems are only available for buprenorphine and fentanyl. In hepatic impairment, fentanyl is safe, but with the exception of codeine and tramadol; other opioids should be used with caution. In renal failure: fentanyl, hydromorphone, and tapentadol are safe. Morphine should be avoided; other opioids should be used with caution. In hemodialysis, buprenorphine, fentanyl, hydromorphone and tramadol (at doses up to $200 \mathrm{mg} /$ day) may be used.

Discussion: Failure to recognize the impact of various situations described throughout this work, including the bioavailability due to oss of oral route, due to pharmacokinetics and pharmacodynamics of the various drugs, either in the context of the impaired metabolism or excretion, or in due to pharmacological interactions, conditions a serious risk of subtreatment of pain and consequent impact in terms of quality of life.

Conclusion: Opioid prescription is safe and effective, even in moderate to severe comorbidities such as renal and hepatic impairment and in patients with no oral route available. In this case, as when considering pharmacological interactions, an individualized therapeutic plan is the best solution and the patient should be assessed regularly. Unadjusted doses may relate to bad pain control and a higher prevalence of adverse events.

Keywords: Analgesics, Opioid/therapeutic use; Cancer Pain/drug therapy; Deglutition Disorders; Hepatic Insufficiency; Neoplasms; Renal Insufficiency

1. Medical Oncology Department. Instituto Português de Oncologia do Porto Francisco Gentil (IPO-PORTO). Porto. Portugal.

2. Molecular Oncology Group. Research Center. Instituto Português de Oncologia do Porto Francisco Gentil (IPO-PORTO). Porto. Portugal.

3. Faculty of Medicine. University of Porto. Porto. Portugal.

4. Internal Medicine Department. Hospital Distrital da Figueira da Foz. Figueira da Foz. Portugal.

5. Unit for the Study and Treatment of Pain. Instituto Português de Oncologia do Porto Francisco Gentil (IPO-PORTO). Porto. Portugal.

$\bowtie$ Autor correspondente: Cláudia Vieira. claudiampvieira@gmail.com

Recebido: 07 de março de 2018 - Aceite: 31 de outubro de 2018 | Copyright @ Ordem dos Médicos 2019 


\section{INTRODUÇÃO}

A Organização Mundial da Saúde (OMS) propôs uma abordagem em pirâmide ou escada, com três degraus para o tratamento farmacológico da dor oncológica. No terceiro nível, com dor mais intensa, a OMS recomenda a utilização de opióides major. ${ }^{1}$ Estudos clínicos não controlados comprovaram que estas orientações são efetivas em $70 \%$ a $100 \%$ dos pacientes com cancro. ${ }^{2}$

A dor em oncologia pode ser causada por efeitos diretos do tumor (por exemplo a invasão do osso, a compressão do nervo...) ou por complicações do tratamento (por exemplo fibrose radiógena, neuropatia induzida pela quimioterapia, a dor do pós-operatório....). ${ }^{3,4}$ Além disso, existe a possibilidade de a dor poder não estar relacionada diretamente com o cancro ou o seu tratamento. ${ }^{5-7}$

A dor tem impacto na qualidade de vida, interferindo a nível do funcionamento físico, psicológico e social (na medida em que altera o sono, o humor e a capacidade de desfrutar a vida); o seu controlo é por isso um objetivo major no tratamento oncológico. ${ }^{8-10}$ Apesar disso, é referenciado que mais de $50 \%$ dos doentes oncológicos em tratamento ativo, mesmo em países desenvolvidos, têm dor não controlada. ${ }^{11}$

Um artigo de revisão de 2016, que analisou revisões sistemáticas e meta-análises em doentes oncológicos estima uma prevalência de dor de $39,3 \%$ após um tratamento curativo, $55,0 \%$ durante o tratamento oncológico e $66,4 \%$ na doença avançada, metastática e em doentes terminais. ${ }^{12} \mathrm{Um}$ artigo com pacientes portugueses, publicado em 2017 sugere taxas de subtratamento da dor oncológica em um quarto dos doentes avaliados. ${ }^{13}$ Este trabalho conclui que o subtratamento da dor oncológica é um problema de saúde pública major. Os mesmos autores, num outro artigo, analisam as barreiras à prescrição de opióides e consequentemente ao ótimo controlo da dor oncológica. ${ }^{14}$

A analgesia farmacológica é parte integrante da estratégia do tratamento antitumoral e paliativo em fases mais avançadas da doença. ${ }^{15} \mathrm{~A}$ morfina e outros analgésicos de ação central são indicados no tratamento da dor crónica moderada a severa, particularmente na dor oncológica. ${ }^{7,16}$ Apesar de universalmente aceite, a prescrição e a aplicação de analgesia são muitas das vezes dificultadas ou mesmo impossibilitadas por condicionantes individuais ${ }^{17}$ que podem ter a ver com comorbilidades e/ou complicações da doença oncológica que condicionam a escolha do analgésico(s) ideal(ais) (de base ou de resgate) ou mesmo adjuvantes e a aplicação dessas orientações. .11,14 $^{11}$

Este trabalho tem como objetivo uma revisão bibliográfica sobre a utilização prática de opióides em algumas situações específicas como perda de via oral, insuficiência renal (IR) e hepática (IH). Serão ainda referenciadas as principais interações farmacológicas dos analgésicos de ação central bem como a utilização de formulações de ação imediata no contexto da dor crónica.

\section{MATERIAL E MÉTODOS}

Neste artigo os autores fizeram uma revisão dos anal- gésicos opióides de ação central, disponíveis em Portugal, em farmácia não hospitalar (segundo prontuário terapêutico português), com indicação no tratamento da dor oncológica e dando particular ênfase àqueles disponíveis em formulações entéricas, transdérmicas (TD) e subcutâneas (SC). ${ }^{18}$ Os fármacos e doses são descritos segundo informação baseada no resumo das características do medicamento (RCM) e no folheto informativo para o doente, informação constante no prontuário terapêutico online (último acesso em janeiro 2018) e pesquisa dos autores (disponibilizada na bibliografia). A designação de marca comercial foi referida pontualmente no sentido de salientar aspetos particulares de determinadas apresentações.

O principal objetivo da pesquisa bibliográfica foi refletir sobre soluções possíveis em caso de perda de via oral, $\mathrm{IH}$ e renal; foram também abordadas algumas interações farmacológicas; são também referidas as formulações de ação imediata disponíveis em Portugal.

Nos últimos anos registámos a existência de formulações diversas de opióides que selecionamos tendo em conta, o perfil farmacocinético e farmacodinâmico, o potencial de interação farmacológica e a conveniência da administração para o doente. . $^{2,19,20}$

Neste trabalho o efeito da idade, da perda ponderal/ síndrome anorexia-caquexia e da insuficiência respiratória não são objeto de revisão dirigida, onde na generalidade se aconselha uma titulação mais cuidada e uma vigilância mais apertada.

\section{RESULTADOS}

O prontuário terapêutico português (Tabela 1) classifica os analgésicos de ação central em opióides agonistas (agonistas dos recetores $\mu$ ), como os alcalóides naturais do ópio (morfina e codeína), os análogos semissintéticos (hidromorfona, oxicodona) e os compostos sintéticos (fentanilo); agonistas-antagonistas mistos; agonistas parciais (buprenorfina); o tramadol como agonista fraco dos recetores opóides $\mu$, contribuindo para o efeito analgésico a potenciação da transmissão serotoninérgica e inibição da recaptação de noradrenalina e o tapentadol que tem um duplo mecanismo de ação, sendo agonista do recetor opióide e inibidor da recaptação de noradrenalina. 18,21,22

Nas Tabelas 1 e 2 encontram-se sumarizados, por ordem alfabética, os analgésicos opióides disponíveis em Portugal, ${ }^{18}$ para a dor oncológica, selecionados segundo os critérios referidos na secção Material e Métodos.

A morfina e o tramadol são os únicos opióides fortes com formulações parentéricas, passíveis de administração por via endovenosa (EV) e subcutânea (SC).

Os efeitos adversos (EA) e as interações medicamentosas são um efeito de classe no que respeita à potenciação do efeito do álcool, hidroxizina, benzodiazepinas e outros depressores do sistema nervoso central (SNC). Existem outros EA que dependem do perfil metabólico; merece aqui particular destaque a via do citocromo $\mathrm{P} 450$, pelo número de fármacos que utilizam esta via metabólica. 
Tabela 1 - Analgésicos opióides: fármacos, formulações disponíveis e advertências $18,21,35,50$

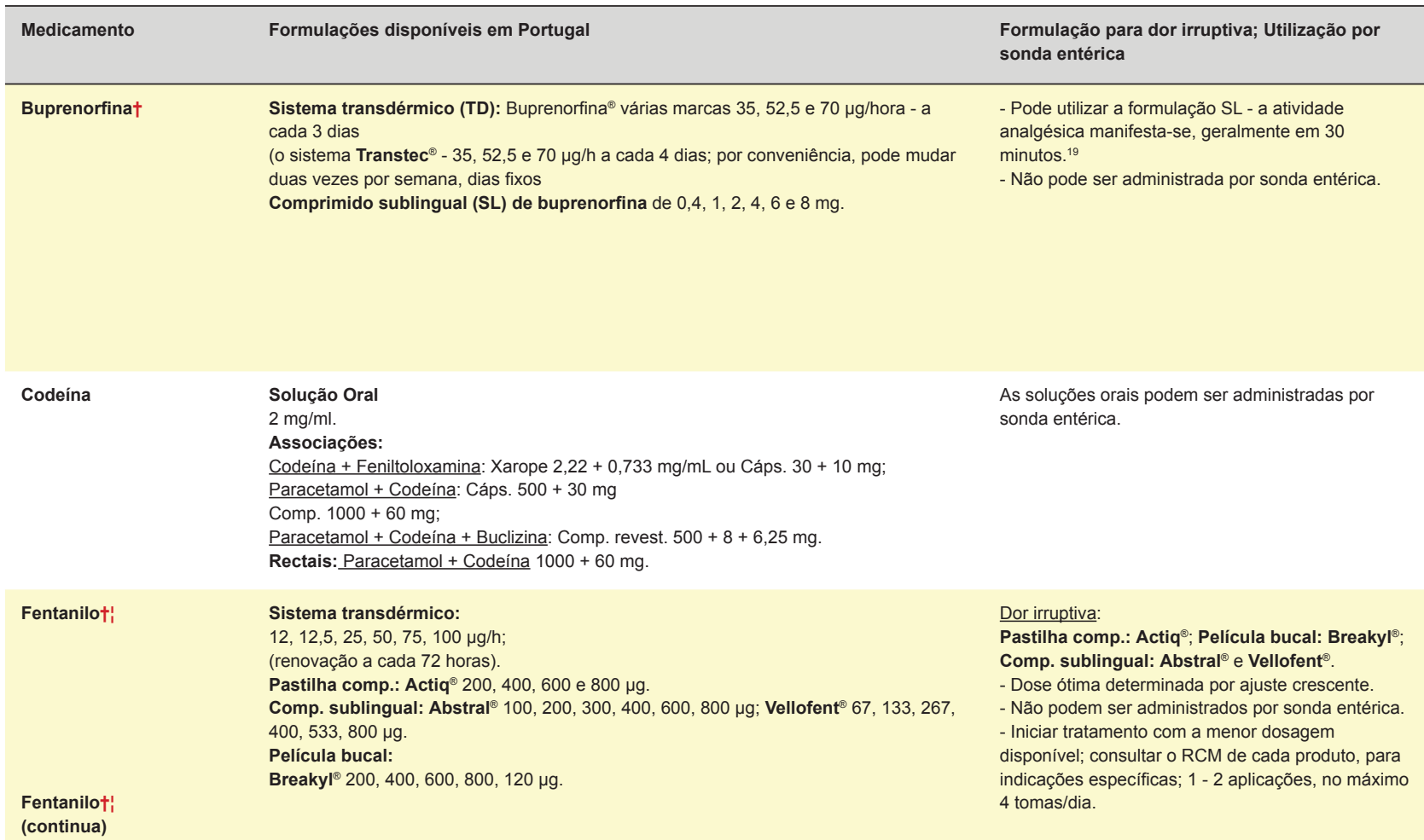

Hidromorfona†¥¥ $\quad$ Comp. libert. prolong.: Jurnista ${ }^{\circledR} 4,8,16,32,64 \mathrm{mg}$

(dose inicial habitual $4 \mathrm{mg} / 24$ horas e titulação em incrementos de 4 a $8 \mathrm{mg} / 24$ horas).
- A formulação para a dor irruptiva (de ação curta) não está disponível em Portugal.

- Não pode ser administrada por sonda entérica.
Morfina

Oxicodona $¥$ /Oxicodona

+ Naloxona

Tapentadol

Tapentadol (continua)

Tramadol
Sol. Oral: Oramorph ${ }^{\circledast}$ 2, 6, $20 \mathrm{mg} / \mathrm{mL}$ (4 gotas $=0,25 \mathrm{~mL}=5 \mathrm{mg}$ de sulfato de morfina). A solução oral tem gosto amargo (pode adicionar-se sumo de laranja ou outro). ${ }^{11}$ Dificuldade na preparação (solução oleosa)

Comp. libert. prolong.: MST ${ }^{\circledR} 10,30,60,100 \mathrm{mg}$ (libertação controlada em 12 horas). Comp. revest. de LI: Sevredol ${ }^{\circledR} 10$ e 20 mg.
Dor irruptiva: Oramorph ${ }^{\circledast}$ (adequada a SNG/ PEG) e comprimidos de ação imediata como Sevredol ${ }^{\circledR}$ (dose igual a $1 / 6$ da dose total de 24 horas; repetida com intervalos de 1 hora). ${ }^{11}$
Oxicodona Comp. libert. prolong. Olbete ${ }^{\circledR} 5,10,20,40,80 \mathrm{mg}$; Oxicodona + naloxona Comp. libert. prolong. Targin ${ }^{\circledast} 5+2,5 \mathrm{mg} ; 10+5 \mathrm{mg} ; 20+10 \mathrm{mg}$.

\begin{abstract}
- A formulação de ação curta não está disponível em Portugal.

- Não pode ser dada por sonda entérica.
\end{abstract}

Comp. libert. prolong.: 50, 100, 150, 200, 250 mg; Início com duas tomas diárias de 50 mg (12/12 horas).

Recomenda-se aumentos de $50 \mathrm{mg} / \mathrm{dia}$, a cada 3 dias.

Gotas orais, sol.: $100 \mathrm{mg} / \mathrm{mL}$.

Comp. 100 mg: Paxilfar ${ }^{\circledR}$.

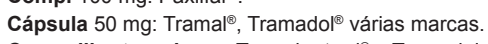

Comp. libert. prolong.: Tramal retard ${ }^{\circledR}$ e Tramadol Actavis ${ }^{\circledR}$ 100, 150, 200 mg; Travex

Long ${ }^{\circledR}$ 150, 200, 300, 400 mg; Tridural $^{\circledR}$ 100, 200, $300 \mathrm{mg}$

Cáps. libert. prolong.: Travex ${ }^{\circledast}$ e Gelotralib ${ }^{\circledast} 50,100,150$ e 200 mg; Tram-u-ron ${ }^{\circledast}$ OD 100, 150 e $200 \mathrm{mg}$.

Solução injectável $50 \mathrm{mg} / \mathrm{mL}$ : várias marcas.
- A formulação de ação curta não está disponível em Portugal.

- Não apresenta formulações passíveis de utilização por sonda entérica.

\section{Dor irruptiva:}

Comp. Orodispersível: Travex Rapid ${ }^{\circledR} 50 \mathrm{mg}$ (podem ser dados por sonda entérica) ou Gotas orais, sol.: $100 \mathrm{mg} / \mathrm{mL}$ (podem ser dadas por sonda entérica).

- As cápsulas de LI podem ser abertas e o seu conteúdo administrado via sonda entérica.

Tramadol +
Dexcetoprofen

Comp. revest. por película: Skudexa ${ }^{\circledR} 75 \mathrm{mg}+25 \mathrm{mg}$

- Não pode ser utilizado por sonda entérica.

Os comprimidos efervescentes podem ser dados por sonda entérica. 
Advertências*

- Pode ser usado em dor neuropática.

- Nos idosos a semivida da buprenorfina não altera de forma significativa.

- Indicação na obstipação refratária. (rotação opióide). ${ }^{28}$

- Risco de prolongamento do intervalo QT com doses acima de $20 \mu \mathrm{g} / \mathrm{hora}^{25}$

- Precaução se função respiratória comprometida (alguns autores defendem ser a $1^{\text {a }}$ escolha nesta circunstância), ${ }^{50}$ hipotiroidismo, mixedema, doença de Addison, caquexia e patologia do sistema nervoso central (SNC).

- Sem efeito imunodepressor (estudos pré-clínicos). ${ }^{51}$

- Para o sistema TD:

a) No início de tratamento considerar tempo até início de ação e absorção após retirada do sistema TD ${ }^{11}$

b) Absorção semelhante no tórax, abdómen e coxas (as áreas devem ser rodadas em cada aplicação):

c) Pode ser cortado na diagonal ou na horizontal. ${ }^{11}$

- Hipersensibilidade à codeína (hipersensibilidade a outros opióides), asma, doença pulmonar obstrutiva crónica (DPOC), depressão respiratória.

- Controvérsia quanto à existência de efeito de teto; dose máxima $240 \mathrm{mg} /$ dia (pela dose cumulativa de paracetamol na associação); se $>360 \mathrm{mg} /$ dia, sugere-se substituição por um opióide forte. ${ }^{11,37}$

- Possibilidade de existirem fenótipos não metabolizadores e consequente ineficácia analgésica. ${ }^{25}$

Nota: Na nossa prática é mais utilizada como antitússico do que como analgésico

- Para o sistema TD:

- Indicação na obstipação refractária (rotação opióide); ${ }^{28,51}$

- $92 \%$ da dose de fentanilo atinge a circulação sistémica inalterada;

- A absorção é semelhante no tórax, abdómen e coxas (as áreas devem ser rodadas em cada aplicação);

- As reações adversas e interações podem observar-se mesmo após remoção do dispositivo;

- No início de tratamento considerar tempo até início de ação e absorção após retirada do sistema TD; ${ }^{11}$

- Sem dose máxima.

Para as formulações de ação imediata:

- Não são intermutáveis; se alterarmos a formulação há que titular de base.

- Toma única diária. 36,53

- Sem dose máxima.

- Absorção no colon; alteração da biodisponibilidade nas síndromes com atraso do trânsito digestivo

- Pode ser utilizada para início de tratamento opióide.

- Sem efeito imunodepressor (estudos pré-clínicos). ${ }^{5}$

- O tratamento deve iniciar-se por morfina de LI: $5 \mathrm{mg}$ se não fazia opióides e $10 \mathrm{mg}$ se fazia (15 mg em doentes com grande massa muscular) e titular de acordo com as necessidades até de $30 / 30$ minutos. ${ }^{11}$

- As preparações orais de ação retardada (ou libertação modificada) têm uma biodisponibilidade muito variável que deve ser verificada caso a caso; são preferíveis para a fase de manutenção (iniciar com 10 a $30 \mathrm{mg}$ de $12 / 12$ horas). ${ }^{11}$

- Pode ser usada em dor neuropática.

- Não existe dose teto (o limite é o controlo da dor ou EA intoleráveis); a maioria dos doentes necessita de $200 \mathrm{mg} / \mathrm{dia}$ ou menos. ${ }^{11}$

- A solução injetável de morfina: $10 \mathrm{mg} / \mathrm{mL}$ ou $20 \mathrm{mg} / \mathrm{mL}$ pode ser usada por via $\mathrm{EV}$, IM, SC, espinhal ou intraventricular.

- Pode ser usado em dor neuropática. ${ }^{47}$

- Em relação à morfina, parece causar menos sedação, delírio, vómito e prurido, mas mais obstipação na forma isolada.

- Menor incidência de obstipação na associação. ${ }^{41}$

- Segundo estudos pré-clínicos está desprovida de efeito imunossupressor. ${ }^{52}$

- Eficácia na dor nociceptiva e neuropática. ${ }^{37}$

- A dose diária máxima recomendada é $500 \mathrm{mg} / \mathrm{dia}$.

- EA mais comuns são as náuseas, vómitos, tonturas e sonolência. ${ }^{37}$

- EA gastrointestinais são mínimos (menor obstipação associada aos efeitos no sistema noradrenérgico). ${ }^{41}$ Precaução em doentes com depressão respiratória, asma, suspeita de íleo paralítico, antecedentes de convulsões.

- A solução oral existe sob duas formas: gotas ou em doseador e a dosagem das gotas é variável conforme a marca (ex.: Tramal ${ }^{\circledR}$ gotas orais, solução 1 atuação completa no doseador $=5$ gotas $=12,5 \mathrm{mg}=0,125 \mathrm{~mL}$ )

- Usa-se na dose de 50 a $100 \mathrm{mg}$ cada 4 a 6 horas.

- As formas de libertação modificada usam-se a cada 8, 12 ou 24 horas.

- Pode ser usado em dor neuropática.

- Pode causar tonturas e algumas das reações adversas típicas dos opióides- hipotensão (também acontece hipertensão), anafilaxia, alucinações, confusão; crises epiléticas (em doentes suscetíveis e/ ou medicados com fármacos que reduzam o limiar para desencadear crises epiléticas).

- A dose máxima recomendada é de $400 \mathrm{mg} / \mathrm{dia}$ se LI ( $300 \mathrm{mg} / \mathrm{d}$ se idosos; risco de convulsões).11,25,37

- Nota: Tram-u-ron ${ }^{\circledR}$ OD e Tridural ${ }^{\circledR} 1$ toma única diária;

Restantes marcas: tomas de 6 a 12 horas (excepto Travex Rapid ${ }^{\circledR}$ - tomas de $4 / 4$ horas).

- Tratamento sintomático de curta duração da dor aguda moderada a grave em adultos.

- Atenção a patologia inflamatória digestiva.

- Atenção à insuficiência renal e cardíaca. ${ }^{11,19,21}$

- Excluir o uso simultâneo de AINES.

- A dose é ajustável à intensidade da dor. Como os comprimidos têm $37,5 \mathrm{mg}$ de tramadol e $325 \mathrm{mg}$ de paracetamol, não se deve ultrapassar a dose de $325 \mathrm{mg} / \mathrm{dia}$ de tramado (8 comprimidos). 


\section{Disfagia/ Perda de via oral}

Os princípios do controlo da dor, definidos em 1986, afirmavam que devem ser respeitados quatro princípios fundamentais: prescrição pela boca, pelo relógio (intervalos regulares, de acordo com o tempo de ação de cada fármaco), pela intensidade e em função do indivíduo. Assim sendo, a via oral deveria ser, sempre que possível, equacionada como via de administração preferencial; entretanto a via TD surgiu também como alternativa, em função das condições do doente, e mais recente a via SC é também utilizada, na inexistência de outras alternativas e face à possibilidade crescente de cuidados de enfermagem no domicílio. A via EV é utilizada quase exclusivamente em ambiente hospi- talar, particularmente no contexto da rotação de opióides. As vias rectal e intramuscular (IM) são uma alternativa em casos muito pontuais. ${ }^{15}$

Em oncologia, a perda de via oral pode resultar de obstrução mecânica (neoplasias da cabeça e pescoço e esófago) ou funcional (síndromes oclusivos altos e baixos com incapacidade para alimentação entérica) ou dos EA dos tratamentos (mucosite e emese). O tratamento dirigido destas entidades é fundamental no manejo da dor, ${ }^{11,16,23} \mathrm{e}$ tem de evoluir a par do tratamento da dor. Várias doenças (não oncológicas) podem afetar a deglutição, como doenças generalizadas da função neuromuscular (p.e. miastenia grave, esclerose lateral amiotrófica, acidente vascular

Tabela 2 - Analgésicos opióides: Interações farmacológicas

\begin{tabular}{|c|c|}
\hline Medicamento & Interações ${ }^{25}$ \\
\hline Buprenorfina & $\begin{array}{l}\text { - Com benzodiazepinas: risco de alteração neurocognitiva, colapso respiratório ou cardiovascular. } \\
\text { - Possível aumento da concentração sérica de buprenorfina com antifúngicos azóis, macrólidos, inibidores da } \\
\text { protéase do VIH e outros potenciadores do CYP3A4. } \\
\text { - Potencial diminuição da concentração sérica de buprenorfina com enzalutamida e dexametasona. } \\
\text { - Risco de prolongamento do intervalo QT com escitalopram, bortezomib, bevacizumab, dasatinib, degarelix, } \\
\text { dolasetrom, doxorrubicina, epirrubicina, fluroquinolonas, granisetrom, lapatinib, metoclopramida, ondansetrom, } \\
\text { pazopanib, ribociclib, sorafenib, sunitib, voriconazol, ziprasidona. }\end{array}$ \\
\hline Codeína & - Pode aumentar o efeito sedativo com hidroxizina ou outros fármacos com ação no SNC. \\
\hline Fentanilo & $\begin{array}{l}\text { - Precaução na associação a inibidores seletivos da recaptação da serotonina (ISRS), inibidores da recaptação } \\
\text { da serotonina e noradrenalina (ISRSN) e inibidores da monoaminoxidase (IMAO) pelo risco de síndrome } \\
\text { serotoninérgico. } \\
\text { - Pode aumentar a concentração de fentanilo com Claritomicina, cobicistat, eritromicina, fluconazol, imatinib, indinavir, } \\
\text { itraconazol, cetoconazol,nelfinavir, nefazodona, posaconazol, ritonavir, saquinavir, voriconazol. } \\
\text { - Potencial diminuição da concentração sérica de fentanilo com enzalutamida e dexametasona. }\end{array}$ \\
\hline Hidromorfona & $\begin{array}{l}\text { - Risco de excitação ou depressão do SNC e de hipo/ hipertensão com IMAO. } \\
\text { - Redução do efeito analgésico e consequente risco de aparecimento de sintomas de abstinência com agonistas/ } \\
\text { antagonistas da morfina. } \\
\text { - Potencial efeito de bloqueio neuromuscular e depressão respiratória com relaxantes musculares. }\end{array}$ \\
\hline Morfina & $\begin{array}{l}\text { - Com IMAO pelos riscos descritos previamente; ranitidina e rifampicina. } \\
\text { - Os depressores do SNC, bloqueadores neuromusculares e agonistas de opióides potenciam efeitos da morfina pelo } \\
\text { que as suas doses devem ser iniciadas e tituladas cuidadosamente. } \\
\text { - A naloxona e antagonistas de opióides (como a buprenorfina) diminuem os efeitos da morfina. }\end{array}$ \\
\hline $\begin{array}{l}\text { Oxicodona /Oxicodona }+ \\
\text { Naloxona }\end{array}$ & $\begin{array}{l}\text { - A inibição do citocromo P450, } 2 \text { D6 e 3A4 não tem relevância clínica; os inibidores potentes de CYP2D6 podem ter } \\
\text { efeito sobre a eliminação da oxicodona. } \\
\text { - Potencial aumento da concentração sérica com buprenorfina e fentanilo. } \\
\text { - Potencial diminuição da concentração sérica com enzalutamida e dexametasona. }\end{array}$ \\
\hline
\end{tabular}

Tapentadol

- Desaconselha-se associação a agonistas/antagonistas opióides mistos (como buprenorfina) e com ISRS (casos isolados de síndrome serotoninérgico).

- Uso de indutores enzimáticos (como rifampicina e fenobarbital) pode diminuir os efeitos do tapentadol.

- Desaconselha o uso concomitante ou 14 dias após a última toma de IMAO pelo risco de acontecimentos cardiovasculares.

- Contraindicada a administração concomitante com cetoconazol e fluconazol pela inibição das vias excretoras de tapentadol e aumento da sua concentração plasmática.

Tramadol

- Contraindicado com IMAO (simultâneo ou até 14 dias da toma destes) e desaconselhado com ISRS e ISRSN pelo risco de síndrome serotoninérgico.

- Redução da concentração plasmática de tramadol com carbamazepina, quinidina e outros indutores; agonistas e antagonistas de opioides (devido ao bloqueio competitivo dos recetores).

- A administração concomitante com antidepressivos tricíclicos, anti-psicóticos, ISRS e ISRSN pode diminuir o limiar de convulsão.

Tramadol + Dexcetoprofeno

- As de ambos os componentes quando usados isoladamente (ver tramadol).

Tramadol + Paracetamol

- As de ambos os componentes quando usados isoladamente. A administração de paracetamol com outros fármacos que aumentam a indução enzimática a nível hepático (como fenitoína e carbamazepina) pode provocar ou agravar lesão hepática, não sendo no entanto necessária redução de dose, apenas vigilância cuidadosa da função hepática.

£: metabolização no CYP3A4; CYP: citocromo P450 
cerebral, doença de Parkinson) e distúrbios da motilidade esofágica, ${ }^{11,15,16,23}$ que podem surgir no contexto da doença oncológica, quer como doenças primárias quer como síndromes paraneoplásicas.

Além disso, alguns fármacos, incluindo opióides, podem agravar os problemas de deglutição/ingestão por via oral, na medida em que podem induzir xerostomia, diminuir a pressão do esfíncter esofágico inferior ou diminuir o peristaltismo digestivo. . $^{11,15,19}$

A Tabela 1 sumariza as diferentes formulações disponíveis para cada fármaco opióide; a solução deverá ser individualizada caso a caso após caracterização da disfagia, comorbilidades e medicação concomitante.

Nas situações em que seja necessária a colocação de uma sonda digestiva nasogástrica (SNG) ou gastrostomia/ jejunostomia percutânea endoscópica (PEG), por exemplo para manter o estado nutricional do doente até melhoria/ resolução da obstrução com o tratamento dirigido à neoplasia, ou para melhorar a tolerância (ex.: mucosite oral da radioterapia a tumores da cabeça e pescoço) poderão ser utilizadas algumas das formulações indicadas para a via oral. ${ }^{16,23}$

Nos doentes com SNG ou PEG é possível administrar os comprimidos efervescentes como paracetamol com ou sem tramadol, ou as soluções orais, como a de morfina, codeína ou tramadol. Os comprimidos de libertação imediata (LI) podem ser esmagados mantendo o perfil de LI. ${ }^{19}$ Nesta situação há que ponderar a biodisponibilidade farmacológica sempre que há estase ou alteração da barreira mucosa. Os comprimidos de libertação retardada não devem ser esmagados, uma vez que se irá alterar a farmacocinética do opióide (passam a ser absorvidos como opióides de ação imediata). A absorção do fármaco nestas formulações pode também ser comprometida em situações de encurtamento do tubo digestivo (por exemplo após enterectomia). ${ }^{19}$

Os sistemas de aplicação TD poderão ser uma solução para os doentes que não podem deglutir ou que têm alteração da biodisponibilidade do fármaco oral, nas situações de má adesão terapêutica ou polimedicação e na rotação de opióides para controlo de EA (alguns autores defendem que os sistemas TD condicionam menos obstipação). ${ }^{11,19}$ Os sistemas TD não devem ser aplicados sobre pele lesada ou depilada e poderá ocorrer absorção acelerada, em caso de febre ou calor externo. Na conversão para o sistema TD é necessário manter a dose de morfina (ou outro analgésico) que se vinha administrando durante 12 a 24 horas, após primeira aplicação. Na retirada, até pelo menos 12 horas após, não deverá ser administrado opióide de ação prolongada. ${ }^{11}$

Segundo a Sociedade Europeia de Oncologia (ESMO), o fentanilo e a buprenorfina TD deverão ser reservados para pacientes cujas necessidades de opióides são estáveis, não sendo os fármacos ideais para titulação de dose. O seu uso é recomendado nos pacientes sem via oral disponível, com má tolerância à morfina ou má adesão ao tratamento analgésico oral. ${ }^{24,25}$ A sua utilização requer monotorização, especialmente nas situações clínicas agu- dizadas, por ex. mucosite, em que a rápida melhoria das lesões poderá condicionar EA por excesso de dose.

O uso de opióides de ação imediata está condicionado às situações de dor irruptiva de acordo com a definição em uso. ${ }^{26} \mathrm{O}$ fármaco idealmente desenhado para a dor irruptiva é o fentanilo, no formato transmucoso, sublingual e película. A morfina injectável também pode ser utilizada, mas as formulações orais de morfina estão indicadas para a dor incidental (que deve ser diferenciada da dor irruptiva). ${ }^{27}$

A via SC apresenta a melhor evidência disponível, quando procurámos uma via alternativa à via oral, com uma revisão sistemática e três ensaios clínicos controlados e randomizados. ${ }^{32} \mathrm{E}$ dado o menor risco de complicações, face à via $\mathrm{EV}$, deve ser a preferida. ${ }^{28}$

$\mathrm{Na}$ perda de via oral, e no que respeita à dor, há que equacionar a necessidade de manter fármacos adjuvantes; esta ponderação será feita caso a caso, em função das condições clínicas e analíticas do doente e da disponibilidade de formulações alternativas; é frequente a necessidade de suspender anti-inflamatórios não esteroides (AINEs), e eventualmente antidepressivos e anticonvulsivantes; para estes últimos equacionar sempre o risco de abstinência.

Nos cuidados de fim de vida, em casos específicos com boa rede social de apoio e possibilidade de cuidados de saúde no domicílio (ou de proximidade), poderá administrar-se tramadol ou morfina SC em ambulatório.

\section{Insuficiência hepática (IH)}

A IH, caracterizada por deterioração grave da função hepática, pode ser devida a doença aguda ou crónica. ${ }^{29,30}$

O impacto da IH na metabolização de fármacos está bem descrito na literatura pela importância que este órgão assume na metabolização de fármacos e pró-fármacos ${ }^{31,32}$; acresce que em situação de ascite, de hipoalbuminémia, a IH pode também condicionar alterações da absorção e distribuição dos fármacos. ${ }^{33}$

Na presença de cirrose associada a gastrite, gastropatia hipertensiva, atraso do esvaziamento gástrico e consequente diminuição da absorção recomendam-se formulações de LI. ${ }^{33}$

A morfina oral sofre um acentuado fenómeno de primeira passagem no fígado, do qual resulta que cerca de $90 \%$ da quantidade que entra em circulação será inativada no fígado por glicuronoconjugação (assim aparecendo na urina). Em doente com IH grave, os efeitos da morfina estão potenciados por diminuição da biotransformação, por diminuição da albumina circulante, quantitativa e qualitativamente (diminuição do volume de distribuição). ${ }^{21}$

Tanto a morfina, como a oxicodona e a hidromorfona são opióides hidrofílicos pelo que devem ser iniciados em menor dose e titulados de forma progressiva e lenta na presença de ascite. A buprenorfina liga-se às frações de $\alpha$-globulina e $\beta$-globulina. ${ }^{34}$ Contudo, estando estas proteínas também diminuídas na cirrose avançada, recomenda-se o tratamento com doses mais baixas de buprenorfina. ${ }^{35,36}$

No que respeita ao metabolismo hepático dos fármacos 
Tabela 3 - Analgésicos opióides e insuficiência hepática $(\mathrm{IH})^{21,35,50}$

\begin{tabular}{|c|c|c|c|c|}
\hline Medicamento & Semi-vida & $\begin{array}{l}\text { Ligação proteínas } \\
\text { plasmáticas }\end{array}$ & Metabolismo hepático & Recomendação \\
\hline Buprenorfina & $37 \mathrm{~h}$ & $\begin{array}{l}96 \% \\
\text { (ligação às alfa e beta } \\
\text { globulinas) }\end{array}$ & CYP450 3A4 & $\begin{array}{l}\text { USAR COM PRECAUÇÃO. } \\
\text { - Sem quantificação da redução de dosagem } \\
\text { necessária. Usar com precaução pela extensa } \\
\text { metabolização hepática. }\end{array}$ \\
\hline Codeína & $4-6$ horas & $7 \%$ & CYP450 2D6 & $\begin{array}{l}\text { NÃO SE RECOMENDA O USO. } \\
\text { - Sem estudos na doença hepática crónica. }\end{array}$ \\
\hline Fentanil TD & 17 horas & $80 \%$ & CYP450 3A4 & $\begin{array}{l}\text { SEGURO. } \\
\text { ^ Child-Pugh A e B: iniciar tratamento com } \\
\text { redução da dose de } 50 \% \text {. } \\
\text { ^ Child-Pugh C: não usar. }\end{array}$ \\
\hline Hidromorfona & 1 - 3 horas & $15 \%$ & Glucoronidação & $\begin{array}{l}\text { USAR COM PRECAUÇÃO. } \\
\text { - Redução da dose em } 25 \% \text { - 50\%. } \\
\text { Titulação lenta. }\end{array}$ \\
\hline Oxicodona & 3 horas & $45 \%$ & CYP450 e $3 A 4$ & USAR COM PRECAUÇÃO. \\
\hline Morfina & $3-12$ horas & $35 \%$ & Glucoronidação & $\begin{array}{l}\text { USAR COM PRECAUÇÃo. } \\
\text { - Redução da dose e frequência em 50\%. } \\
\text { - Titulação de dose lenta. }\end{array}$ \\
\hline Tapentadol & 6 horas & $20 \%$ & $\begin{array}{l}\text { Principalmente glucuronidação e, } \\
\text { em menor grau, por CYP2C9 e } \\
\text { CYP2C19 }\end{array}$ & $\begin{array}{l}\text { USAR COM PRECAUÇÃO. } \\
\text { - Redução da dose na IH moderada (utilizar dose } \\
\text { diária máxima inferior a } 600 \text { mg se libertação } \\
\text { prolongada). }{ }^{25}\end{array}$ \\
\hline Tramadol & 7 horas & $20 \%$ & $\begin{array}{l}\text { CYP450 2D6 e 3A4 e } \\
\text { glucoronidação }\end{array}$ & $\begin{array}{l}\text { NÃO SE RECOMENDA O USO. } \\
\text { LI: } \\
\text { * Poderá usar-se } 50 \text { mg cada } 12 \text { horas. Alguns } \\
\text { estudos referem } 25 \text { mg cada } 8 \text { horas. } \\
\text { Libertação prolongada: } \\
\text { * Child-Pugh A e B usar com precaução (risco } \\
\text { de acumulação). } \\
\text { ^ Child-Pugh C: não usar. }\end{array}$ \\
\hline
\end{tabular}

CYP: citocromo P450

é importante ter em conta o cálculo do rácio de extração hepática, definido na literatura de cada fármaco. Considera-se um rácio de extração hepática de 0 quando o fígado é incapaz de metabolizar o fármaco e de 1 quando todo o fármaco é metabolizado na primeira passagem. Dando como exemplos, a morfina e fentanilo têm rácio $>0,7$ (rácio de extração alto) e a metadona um rácio < 0,3 (rácio de extração baixo). Em situações de shunt porto-sistémico a diminuição da capacidade metabólica hepática poderá aumentar a biodisponibilidade do fármaco se este tiver um elevado rácio de extração hepática. ${ }^{35}$

A codeína é absorvida por via oral e a sua eliminação ocorre quase exclusivamente por biodegradação hepática em metabólitos inativos excretados na urina (apenas 10\% sofre desmetilação em morfina) ${ }^{21}$; o seu uso na IH é contraindicado.

Nos casos de IH (também é válido para a IR e para pacientes idosos) deve efetuar-se uma titulação lenta da morfina e iniciar com doses baixas (ex.: início por $5 \mathrm{mg}$ de morfina de LI) e aumentar-se o intervalo entre doses das habituais 4 horas, para 6 horas. Contudo devem permitir-se doses de resgate, para os picos de dor (mesma dose até ao intervalo mínimo de 1 hora). Passadas 24 a 48 horas, as necessidades diárias devem ser reavaliadas e a dose diária fixa ajustada, até que se obtenha um controlo satisfatório da dor, sem EA significativos. Uma vez controlada a dor, faz-se a manutenção, com uma fórmula de libertação prolongada mais cómoda (a dose diária é sobreponível à da morfina de LI) ou outro opióide em dose equivalente. ${ }^{11,21}$

À medida que aumenta o compromisso metabólico secundário à disfunção hepática, também se altera o perfil farmacocinético individual dos vários opióides; a redução de dose para a hidromorfona, o aumento do intervalo entre administrações e redução de dose para o tramadol, tapentadol, morfina e oxicodona; a codeína deverá ser evitada. ${ }^{30,32,37}$

A Tabela 3 resume as recomendações e indicações dos opióides no caso de $\mathrm{IH}$.

\section{Insuficiência renal (IR)}

A doença renal crónica define-se como a alteração da função ou estrutura renal com mais de três meses de 

Indicação
Notas

Risco de toxicidade hepática.

Metabolizada maioritariamente em morfina no fígado.

Sem alterações da farmacocinética.
Pode ter a eficácia reduzida, por menor biotransformação do fármaco no seu metabolito ativo (morfina).

Pode acumular-se em administrações repetidas devido a ligação a proteínas tecidulares.

Risco de overdose (rácio de extração intermédio).

Iniciar tratamento com redução da dose (33\% - 50\%).

Risco de overdose (rácio de extração alto).

Potencial aumento da biodisponibilidade da morfina oral até $200 \%$ na falência hepática.

Desaconselha-se a sua prescrição em doentes com $\mathrm{IH}$ grave. ${ }^{25}$
Início de ação e eficácia variáveis.

Aumento da semivida até 2 vezes.

Acumulação de metabólitos com potencial tóxico grave: depressão respiratória, tolerância analgésica, neurotoxicidade.
Concentrações séricas máximas da formulação de libertação sustentada são atingidas em 3 - 6 horas.

Alterações da farmacocinética na IH moderada a severa (risco de convulsões). ${ }^{25}$

evolução ${ }^{38}$ sendo a sua gravidade estratificada através da clearance de creatinina; considera-se que uma clearance abaixo de $30 \mathrm{~mL} / \mathrm{min}$ traduz IR e abaixo de $15 \mathrm{~mL} / \mathrm{min}$ traduz falência renal terminal com necessidade de avaliar indicação para suporte dialítico. O conhecimento da farmacocinética e farmacodinâmica dos opióides na doença renal é escasso. ${ }^{38}$ Nos doentes sob tratamento dialítico na escolha do fármaco devem ser ponderados as características do fármaco e o tipo de técnica dialítica usada, as propriedades físicas da diálise (nomeadamente tamanho dos poros do filtro), o débito e a eficácia da técnica. ${ }^{31}$

São reconhecidas várias lacunas no conhecimento da farmacocinética da maioria dos opióides na IR; existem poucos ensaios randomizados, sem seguimento a longo prazo e muitos fatores de viés, para além do efeito da IR propriamente dita. Dado não existir qualquer estudo prospetivo, com um bom nível de evidência, recomenda-se que todos os fármacos sejam utilizados com vigilância frequente e adequada. Contudo a presença de IR não é razão para atrasar a introdução de um opióide quando o mesmo é necessário para controlar a dor, particularmente oncológica. ${ }^{39}$
Existem orientações internacionais sobre cada opióide e as doses seguras para os diferentes níveis de IR, cabendo ao médico, a titulação adequada. ${ }^{40}$

Para uma taxa de filtração glomerular estimada (TFG) 30 - $89 \mathrm{~mL} / \mathrm{min}$ (IR leve a moderada) pode ser considerado tratamento com quase todos os opióides (com indicação na dor oncológica), com eventual ajuste de dose e frequência. A função renal deverá ser monitorizada e o tratamento alterado se registo de deterioração, não esquecendo a correção de fatores reversíveis da função renal e a ponderação de fatores de confundimento tais como a caquexia, hipoalbuminémia, edemas ou agudização da IR crónica. ${ }^{39}$

Um documento de consenso recente promovido pela European Federation of IASP Chapters (EFIC) conclui que com base nos dados farmacocinéticos disponíveis, os opióides com menor potencial de dano nos pacientes com insuficiência real são o fentanilo e a buprenorfina. A hidromorfona e a oxicodona poderão ser utilizadas com vigilância mais apertada. A Codeína, a morfina e o tramadol deverão ser evitados. ${ }^{41}$

A Tabela 4 resume as recomendações conhecidas para 

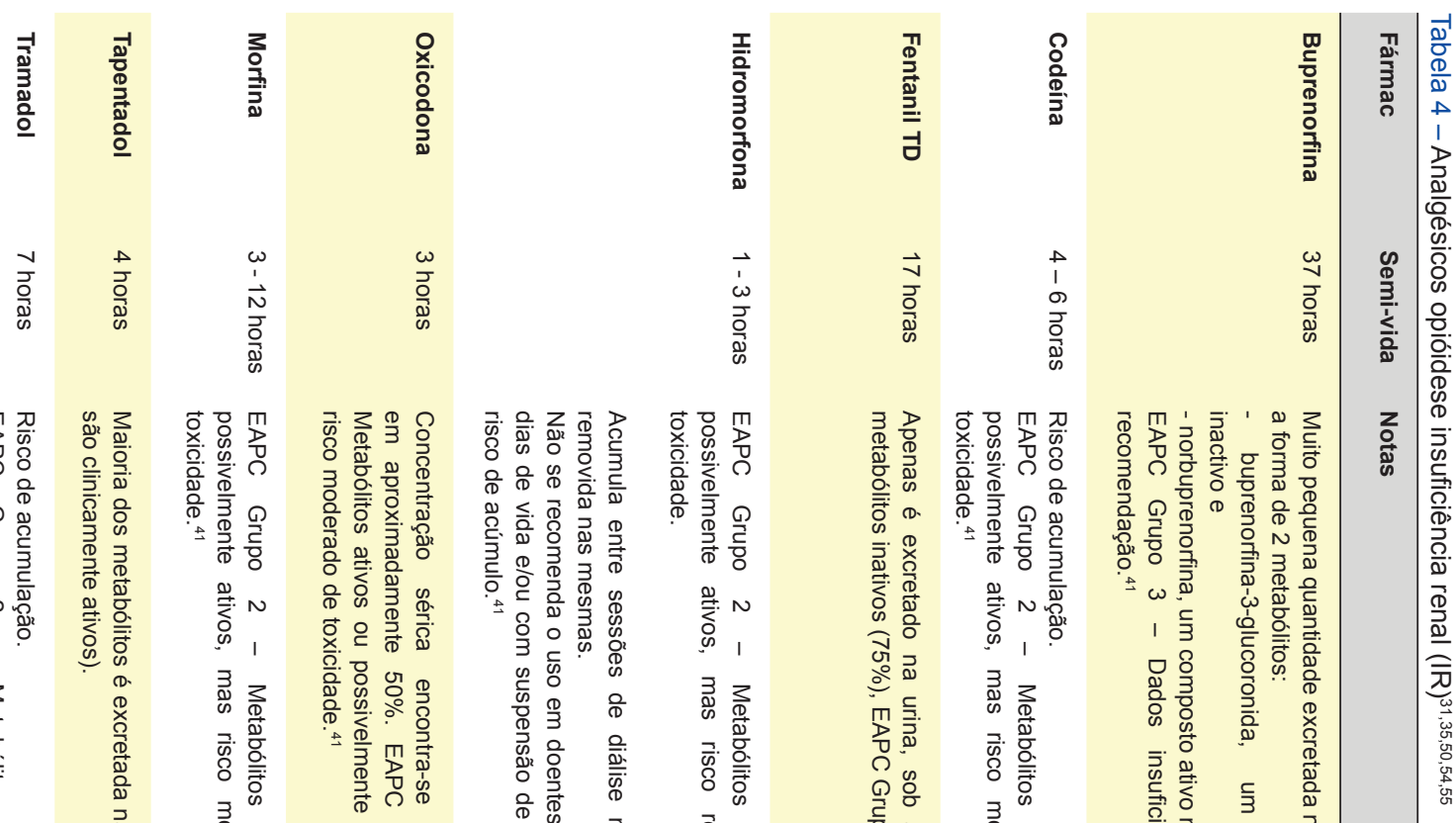

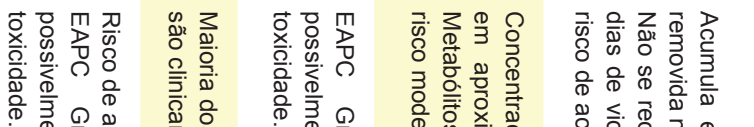

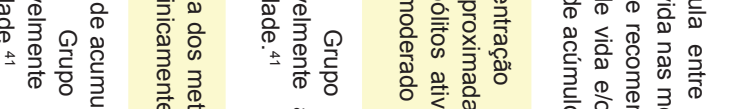

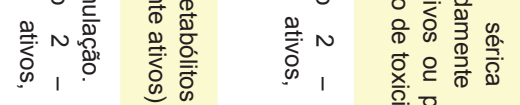

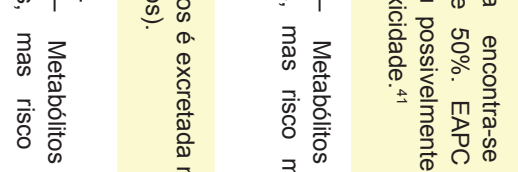

帝

उำ

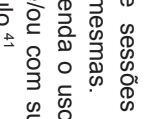

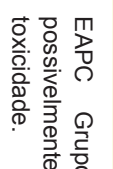

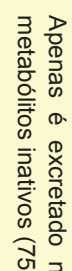

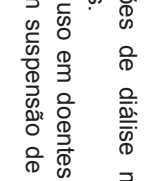

䓛

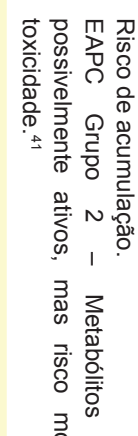

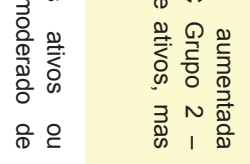

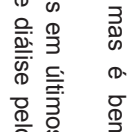

융

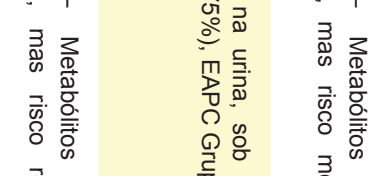

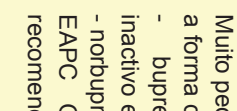

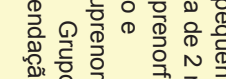

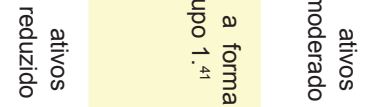

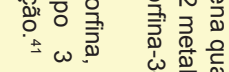

ㅇํㅇ ำ

कㅇ

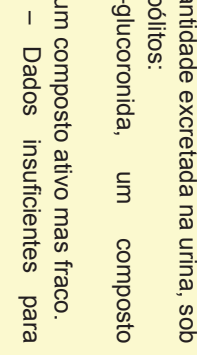

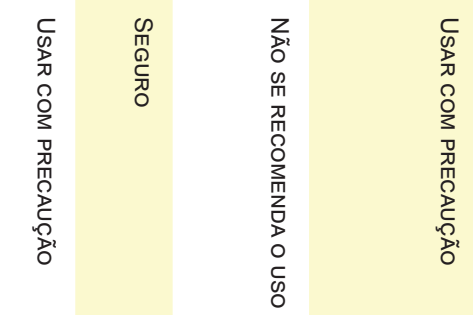

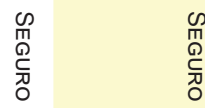
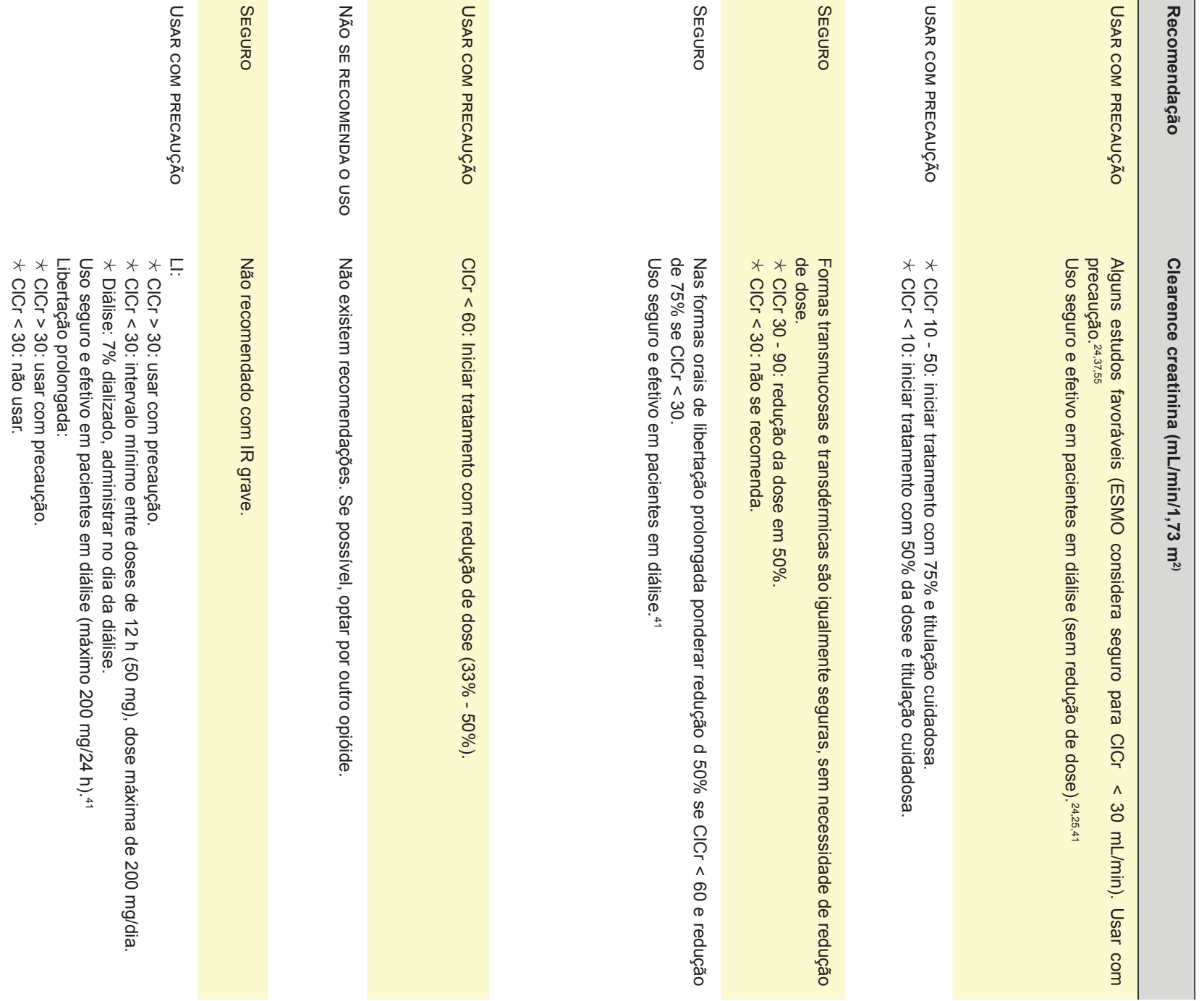
os opióides na presença de IR. ${ }^{42}$

\section{DISCUSSÃO}

No ato de prescrição de um opióide as regras universais de segurança devem estar presentes: diagnóstico cuidadoso; avaliação psicológica; consentimento informado; avaliação pré e pós-intervenção da intensidade e impacto funcional da dor; titulação apropriada do opióide instituído e/ou medicação coadjuvante; reavaliação ao longo do tempo seguindo a regra dos 4 As (analgesia; atividades da vida diária; EA; comportamento aberrante); revisão periódica do diagnóstico de dor e comorbilidades; documentação adequada e auditável no processo clínico da avaliação da dor e consumo de fármacos analgésicos (entre outras). ${ }^{43-45}$

A titulação correta do fármaco, o ensino de medidas preventivas dos EA e a rotação de opióides sempre que necessário, ajudam no controlo da dor e na gestão dos eventos adversos. ${ }^{18}$

A prescrição de procedimentos invasivos, anestésicos ou neurocirúrgicos, pode ser uma opção em doentes que não tenham respondido de forma adequada à medicação instituída ou que tenham EA não toleráveis. ${ }^{46}$

Os pacientes em tratamento sintomático apresentam, além da dor, inúmeros outros sintomas, que influenciam o seu bem-estar. ${ }^{47}$ Náuseas, astenia, fadiga, obstipação são alguns dos vários sintomas referidos ${ }^{48}$; que além de interagir com dor, influenciam a vivência da mesma, pelo que podem condicionar menor eficácia da analgesia. ${ }^{49}$

O prontuário terapêutico português apresenta algumas lacunas, quando comparado com outros países europeus. O fato de não estarem disponíveis em Portugal, o fentanilo aerossol nasal, as cápsulas de sulfato de morfina cujos grânulos podem ser administrados por sondas, as formulações de libertação imediata da hidromorfona ou tapentadol, representa uma dificuldade acrescida no momento da prescrição.

A aplicação de formulações de ação imediata e as interações farmacológicas colocam dúvidas a muitos médicos mas foram abordadas muito sumariamente neste artigo. $\mathrm{O}$ efeito da idade, da perda ponderal/síndrome anorexia-caquexia e da insuficiência respiratória não foram objeto desta revisão dirigida. E também não foi dado particular enfoque as síndromes de dor oncológica específicas que necessitam de tratamento dirigido, como é o caso, por exemplo, da dor neuropática por infiltração/compressão nervosas ou a dor associada a um quadro suboclusivo visceral ou a situações de uso inadequado de fármacos por condicionantes biológicas individuais que alteram a farmacocinética e/ou a farmacodinâmica dos medicamentos prescritos. A falta destes temas representa uma limitação mas também uma oportunidade de investigação e artigos futuros.

Outra limitação deste artigo de revisão é a antiguidade de algumas citações, refletindo a antiguidade de algumas ferramentas, artigos e manuais que utilizamos na prática clínica. A necessidade de ensaios clínicos randomizados na área da dor e estudos prospetivos multicêntricos portugueses permitirão que no futuro o nível de evidência do tratamento da dor oncológica seja superior.

\section{CONCLUSÃO}

$\mathrm{Na}$ literatura, o opióide de primeira escolha para a dor oncológica moderada a grave continua a ser a morfina. ${ }^{46}$ A administração oral é a via preferencial. O fentanilo ou buprenorfina TD são recomendados para os pacientes em que a necessidade de opióides é conhecida e estável. São também recomendados nos pacientes com dificuldades na deglutição, baixa tolerância à morfina ou baixa adesão. Pacientes com dor severa, com necessidade de alívio urgente, devem ser tratados como situação urgente, com opióides por via SC ou EV, de acordo com a situação clínica do doente. ${ }^{20,46}$

O não reconhecimento do impacto das várias situações descritas ao longo deste trabalho, nomeadamente a alteração da biodisponibilidade por perda de via oral, por alteração da farmacocinética e farmacodinâmica dos vários fármacos, quer no contexto da insuficiência de órgão responsável pelo metabolismo ou excreção, quer no contexto das interações farmacológicas, condiciona um enorme risco de subtratamento da dor e consequente impacto em termos de qualidade de vida.

\section{PROTECÇÃO DE PESSOAS E ANIMAIS}

Os autores declaram que os procedimentos seguidos estavam de acordo com os regulamentos estabelecidos pelos responsáveis da Comissão de Investigação Clínica e Ética e de acordo com a Declaração de Helsínquia da Associação Médica Mundial.

\section{CONFIDENCIALIDADE DOS DADOS}

Os autores declaram ter seguido os protocolos do seu centro de trabalho acerca da publicação de dados.

\section{CONFLITOS DE INTERESSE}

Cláudia Vieira declara ter recebido apoio de F. Hoffmann-La Roche Ltd, MSD, Novartis, Phizer, Astrazeneca, Merck Serono, Grünenthal S.A. e Laboratórios Vitória, sem relação com este artigo.

Marta Brás declara não ter quaisquer conflitos de interesse.

Maria Fragoso declara ter recebido apoio, embora sem conflito de interesse com o presente trabalho, da Grünenthal S.A. e dos Laboratórios Pfizer, Vitória e Menarini.

\section{FONTES DE FINANCIAMENTO}

Este trabalho não recebeu qualquer tipo de suporte financeiro de nenhuma entidade no domínio público ou privado. 


\section{REFERENCIAS}

1. World Health Organization. Cancer pain relief and palliative care. Geneve: WHO; 1996.

2. Jadad AR, Browman GP. The WHO analgesic ladder for cancer pain management. Stepping up the quality of its evaluation. JAMA. 1995;274:1870-3

3. Alcock MM. Defining pain: past, present, and future. Pain. 2017;158:7612 .

4. Marcus NJ. Pain in cancer patients unrelated to the cancer or treatment. Cancer Invest. 2005;23:84-93.

5. Williams AC, Craig KD. Updating the definition of pain. Pain. 2016;157:2420-3.

6. Direcção-Geral da Saúde. Circular Normativa: A dor como $5^{\circ}$ sinal vital. Registo sistemático da intensidade da Dor. Lisboa: DGS; 2003.

7. Direcção-Geral da Saúde. Circular Normativa: Utilização dos medicamentos opióides fortes na dor crónica não oncológica. Lisboa: DGS; 2008 .

8. Christensen HN, Olsson U, From J, Breivik H. Opioid-induced constipation, use of laxatives, and health-related quality of life. Scand $J$ Pain. 2016;11:104-10.

9. von Moos R, Costa L, Ripamonti Cl, Niepel D, Santini D. Improving quality of life in patients with advanced cancer: Targeting metastatic bone pain. Eur J Cancer. 2017;71:80-94.

10. Pereira S, Fontes F, Sonin T, Dias T, Fragosos M, Castro-Lopes J, et al. Neuropathic pain after breast cancer treatment: characterization and risk factors. J Pain Symptom Manage. 2017;54:877-88

11. Gonçalves F. Controlo de sintomas no cancro avançado. Porto: Fundação Calouste Gulbenkian; 2002.

12. van den Beuken-van Everdingen $M H$, Hochstenbach LM, Joosten EA Tjan-Heijnen VC, Janssen DJ. Update on prevalence of pain in patients with cancer: systematic review and meta-analysis. J Pain Symptom Manage. 2016;51:1070-90.

13. Reis-Pina P, Lawlor PG, Barbosa A. Adequacy of cancer-related pain management and predictors of undertreatment at referral to a pain clinic. J Pain Research. 2017;10:2097-107.

14. Reis-Pina P, Lawlor PG, Barbosa A. Cancer-related pain management and the optimal use of opioids. Acta Med Port. 2015;28:376-81.

15. Fernandes JP, Barradas A, Custódio MP, Gago T, Quintela A, Rodrigues $\mathrm{R}$, et al. Manual de terapêutica médica - hematologia e oncologia. Lisboa: Lidel; 2009.

16. Pentheroudakis G, Sessa C, Steering Committee Members. ESMO Pocket Guidelines - Supportive Care. Viganello-Lugano: ESMO; 2016.

17. Nielsen LM, Olesen AE, Branford R, Christrup LL, Sato H, Drewes AM. Association between human pain-related genotypes and variability in opioid analgesia: an updated review. Pain Pract. 2015;15:580-94.

18. Prontuário Terapêutico (Formato: Online). Lisboa: INFARMED; 2016 [consultado em 2018 jan 28]. Disponível em: www.infarmed.pt/web/ infarmed/institucional/documentacao.../prontuario-terapeutico.

19. Ritto C, Naves F, Rocha FD, Costa I, Diniz L Raposo MB, et al. Manual de dor crónica. 2. ${ }^{a}$ ed. Lisboa: Fundação Grünenthal; 2017. p.123-51, 155-98, 229-38 e 315.

20. Jost L, Roila F, Group EGW. Management of cancer pain: ESMO clinical recommendations. AnnOncol. 2008;19:ii119-21.

21. Garrett J, Osswald W, Guimarães S, Moura D, Soares da Silva P Castro Lopes J, et al. Terapêutica medicamentosa e suas bases farmacológicas. Vol I. Porto: Porto Editora; 1999.

22. Faria J, Barbosa J, Moreira R, Queiros O, Carvalho F, Dinis-Oliveira RJ. Comparative pharmacology and toxicology of tramadol and tapentadol. Eur J Pain. 2018;22:827-44

23. Pereira D, Vieira C, Ferreira M. Tratamento de suporte em oncologia. $1 .^{a}$ edição. Porto: MSD; 2015.

24. Ripamonti Cl, Santini D, Maranzano E, Berti M, Roila F, Group EGW. Management of cancer pain: ESMO Clinical Practice Guidelines. Ann Oncol. 2012;23:vii139-54.

25. National Comprehensive Cancer Network. Adult cancer pain. Version 1. 2018. [consultado em 2018 jan 28]. Disponível em: http://www.nccn.org/ professionals/physician_gls/pdf/pain.pdf.

26. Davies PS. Pharmacologic pain management at the end of life. Nurse Pract. 2016;41:26-37.

27. Reis Pina P. Controlo da dor em cuidados paliativos. In: Barbosa A, Reis Pina P, Tavares F, Neto IG, Eds. Manual de Cuidados Paliativos. $3^{a}$ ed. Lisboa: FMUL; 2016. p. 74-8.

28. Radbruch L, Trottenberg P, Elsner F, Kaasa S, Caraceni A. Systematic review of the role of alternative application routes for opioid treatment for moderate to severe cancer pain: an EPCRC opioid guidelines project.
Palliat Med. 2011;25:578-96.

29. Fauci AS, Kasper DL, Hauser SL, Longo DL, Jameson JL, Braunwald E, et al. Harrison's Principles of Internal Medicine. $16^{\text {th }}$ ed. New York: McGraw-Hill; 2008.

30. Lee WM, Stravitz RT, Larson AM. Introduction to the revised American Association for the Study of Liver Diseases Position Paper on acute liver failure 2011. Hepatol. 2012;55:965-7.

31. Johnson SJ. Opioid safety in patients with renal or hepatic dysfunction. Update em 2007 [consultado 2018 jan 28]. Disponível em: ttp:// paincommunity.org/blog/wp-content/uploads/Opioids-Renal-HepaticDysfunction.pdf

32. Chandok N, Watt KD. Pain management in the cirrhotic patient: the clinical challenge. Mayo Clin Proc. 2010;85:451-8.

33. Hamilton JP. Management of pain in patients with advanced chronic liver disease or cirrhosis. 2016. [consultado 2018 jan 28]. Disponível em: www.uptodate.com/contents/management-of-pain-in-patients-withadvanced-chronic-liver-diasease-or-chirrhosis.

34. Heel RC, Brogden RN, Speight TM, Avery GS. Buprenorphine: a review of its pharmacological properties and therapeutic efficacy. Drugs. 1979;17:81-110

35. Buprenorphine/ Codeine/ Fentanyl/ Hydromorphone/ Morphine/ Oxycodone/ Tapentadol/ Tramadol: Drug information. Massachusetts: UpToDate. [consultado 2018 jan 28]. Disponível em: https://www.uptodate.com/contents/buprenorphine-and-naloxone-drug-information? search=Buprenorphine $\% 20$ drug $\% 20$ information\&source=search result\&selectedTitle=2 148

36. Mercadante $\mathrm{S}$. The use of high doses of OROS hydromorphone in an acute palliative care unit. Curr Med ResOpin. 2011;27:2373-6.

37. Cardoso A. Dlssertação: controlo da dor em pacientes oncológicos. Porto: Instituto de Ciências Biomédicas Abel Salazar; 2014

38. Lamb EJ, Levey AS, Stevens PE. The Kidney Disease Improving Global Outcomes (KDIGO) guideline update for chronic kidney disease: evolution not revolution. ClinChem. 2013;59:462-5.

39. King S, Forbes K, Hanks GW, Ferro CJ, Chambers EJ. A systematic review of the use of opioid medication for those with moderate to severe cancer pain and renal impairment: a European Palliative Care Research Collaborative opioid guidelines project. Pall Med. 2011;25:525-52.

40. Hennemann-Krause L. Aspectos práticos da prescrição de analgésicos na dor do câncer. Revista do Hospital Universitário Pedro Ernesto. 2012;11:1-16

41. O'Brien T, Christrup LL, Drewes AM, Fallon MT, Kress HG, McQuay HJ, et al. European Pain Federation position paper on appropriate opioid use in chronic pain management. Eur JPain. 2017;21:3-19.

42. Cockcroft DW, Gault MH. Reduction of creatinine clearance from serum creatinine. Nephron 16:31-41, 1976

43. Argoff CE, Silvershein DI. A comparison of long- and short-acting opioids for the treatment of chronic noncancer pain: tailoring therapy to meet patient needs. Mayo Clin Proc. 2009;84:602-12.

44. Gourlay DL, Heit HA, Almahrezi A. Universal precautions in pain medicine: a rational approach to the treatment of chronic pain. Pain Med. 2005;6:107-12.

45. Coluzzi F, Taylor R Jr, Pergolizzi JV Jr, Mattia C, Raffa RB. Good clinical practice guide for opioids in pain management: the three Ts - titration (trial), tweaking (tailoring), transition (tapering). Rev Bras Anestesiol. 2016;66:310-7.

46. Hanks GW, Conno F, Cherny N, Hanna M, Kalso E, McQuay HJ and others members of the Expert Working Group of the Research Network of the European Association for Palliative Care. Morphine and alternative opioids in cancer pain: the EAPC recommendations. $\mathrm{Br}$ Cancer. 2001;84:587-93.

47. Walsh D. Pharmacological management of cancer pain. Sem Oncol. 2000;27:45-63.

48. Portenoy RK, Thaler HT, Kornblith AB, Lepore JM, Friedlander-Klar H Coyle N, et al. Symptom prevalence, characteristics and distress in a cancer population. Qual Life Res.1994;3:183-9.

49. Sutton LM, Porter LS, Keefe FJ. Cancer pain at the end of life: a biopsychosocial perspective. Pain. 2002;99:5-10.

50. Pergolizzi J, Böger RH, Budd K, Dahan A, Erdine S, Hans G, et al. Opioids and the management of chronic severe pain in the elderly: consensus statement of an International Expert Panel with focus on the six clinically most often used World Health Organization Step III opioids (buprenorphine, fentanyl, hydromorphone, methadone, morphine, oxycodone). Pain Pract. 2008;8:287-313.

51. BC Guidelines.ca. Palliative care for the patient with incurable cance 
or advanced disease - part 2: pain and symptom management. 2017. [consultado 2018 jan 28]. Disponível em: https://www2.gov.bc.ca/ gov/content/health/practitioner-professional-resources/bc-guidelines/ palliative-pain-management.

52. Sacerdote P. Opioids and the immune system. Palliat Med. 2006;20:s915.

53. Weinstein SM. A new extended release formulation (OROS) of hydromorphone in the management of pain. Ther Clin Risk Manag. 2009;5:75-80.

54. Davison. Management of chronic pain in chronic kidney disease.
Massachusetts: UpToDate. 2016. [consultado 2018 jan 28]. Disponível em: www.uptodate.com/contents/management-of-chronic-pain-inchronic-kidney-disease.

55. Davison SN, Moss AH. Supportive care: meeting the needs of patients with advanced chronic kidney disease. Clin J Am Soc Nephrol. 2016;11:1879-80.

56. Koppert W, Filitz J, Tröster A, Ihmsen H, Angst M, Flor H, et al. Activation of naloxone-sensitive and -insensitive inhibitory systems in a human pain model. JPain. 2005;6:757-64. 